Z. Klin. Chem. Klin. Biochem.

12. Jg. 1974, S. 93-97

\title{
Biochemische Stigmata menschlicher Hautoberfläche im Alter
}

\author{
Von E. Schwarz \\ Hautklinik im Klinikum Steglitz der Freien Universität Berlin
}

(Eingegangen am 18. Oktober 1973)

\begin{abstract}
Altersveränderungen der oberflächlichen Hornschicht wurden hinsichtlich des Gehaltes und der Zusammensetzung der freien Aminosäuren, von Harnstoff, Ammoniak, Urocanin-, Pyrrolidoncarbon- und Milchsäure untersucht. Signifikante Vermehrungen fanden sich im Mittel für die freien Aminosäuren insgesamt, für Glycin, $\alpha$-Alanin und Valin sowie Verminderungen für Threonin, Glutaminsäure, Histidin, Gitrullin, Harnstoff und Milchsäure. Die auffällige Minderung der beiden letztgenannten Substanzen ist offenbar auf reduzierte Schweißbildung im Alter zurückzuführen. Die mögliche Bedeutung der Befunde für das Oberflächen-Milieu der Altershaut wird diskutiert.
\end{abstract}

\section{Biochemical stigmas of human skin surface in old age}

Age-dependent changes in the superficial horny layer of human skin were investigated with respect to the quantity and type of free amino acids, and the quantities of urea, ammonia, urocanic-, pyrrolidone carboxylic acid and lactic acid. Significant increases were found for the total free amino acids, for glycine, $\alpha$-alanine and valine, as well as decreases for threonine, glutamic acid, histidine, citrulline, urea and lactic acid. The striking diminution of the two last substances is apparently due to reduced sweating in the old. The possible significance of these results for the behaviour of the skin surface, in old age is discussed.

Die wasserlöslichen, kleinmolekularen Verbindungen der Hornschicht (das ,Wasserlösliche") (1-10) sind wesentlich für Schutzfunktionen der Haut gegenüber der Umwelt verantwortlich, die durch Begriffe wie Säuremantel $(11,12)$, Pufferkapazität $(13,14)$, Hygroskopizität $(5$, $6,15)$ und Bactericidie $(16,17)$ charakterisiert sind. Zivilisationsschäden der Haut beruhen nicht selten auf Auslaugung des Wasserlöslichen.

Als Quelle des Wasserlöslichen sind einmal Schweißrückstände (etwa 10-30\% (5)), vor allem aber die epidermalen Keratinisationsprozesse anzusehen.

Die vielfältigen Ausprägungen epithelialer Zellverhornung (Haare, Nägel usw.) umfassen in der Epidermis nicht nur die Bildung besonderer Strukturen ( $\mathbf{z}$.

B. Hornzellmembranen, Keratohyalin) und spezieller (Sklero-) Proteine (z. B. Tonofibrillen, „Matrix“-Protein), die dem Keratin-Begriff im klassischen Sinn (18) nicht entsprechen, sondern auch die Destruktion von Zellstrukturen wie Zellkernen und -organellen sowie den Um- und/oder Abbau von Zellproteinen (19).

Autoradiographisch lassen sich zwei Schwerpunkte der Proteinsynthesen zeigen, wobei die einzelnen Aminosäuren mit untetschiedlicher Präferenz inkorporiert werden: Leu, Phe; Val, Lys und Met im Stratum basale und Gly, Ser, His, Arg und Cys im Stratum granulosum $(20,21)$. Aus der Epidermis wurden Proteine isoliert, in denen jeweils meist eine Aminosäure prävalierte: ein Gly-reiches Protein (22), ein His-Protein $(22,23)$, ein Cys-Protein $(24,25)$, ein Tyr-Protein (26) und ein Ser-reiches Protein (27). Für die Hornzellmembranen ist der Pro-Reichtum herausgestellt worden $(28,29)$. Aus alkali-löslichen Proteinen (30) lassen sich anscheinend leicht Peptide freisetzen, die zu etwa $\$ 0 \%$ aus Ser und Gly bestehen.

Als Uberbleibsel („Fossilien“ $(7,8)$ ) der Keratinisation gestatten die freien Aminosäuren und zugehörige Verbindungen gewisse Rückschlüsse auf Vorgänge derselben resp. ihre Störungen. Pathologische Verhornungsprodukte wie Psoriasis- (31) und Ichthyosisschuppen sowie Callus (32) ließen typische Abweichungen gegenüber der charakteristischen Zusammensetzung des Wasserlöslichen normaler Hornschicht erkennen. Auch in der Hornschicht klinisch unauffälliger Haut bei Neurodermitis (33) und mikrobiellem Ekzem (34) waren Differenzen zu finden, nicht eindeutig dagegen in unbefallener Psoriatiker-Haut (35).

Als altersbedingte Veränderungen des Wasserlöslichen wurden Verminderung von Harnstoff und Glu bzw. Vermehrung von Val und Urocaninsäure gefunden (36).

- Eine Erweiterung der Analysenreihe und zusätzliche Bestimmung anderer Substanzen des Wasserlöslichen wie Lactat und Pyrrolidoncarbonsäure sowie Prüfung von Korrelationen verschiedener Komponenten des Wasserlöslichen sollten zu Fragen ihrer Herkunft und der Natur der Veränderungen Aufschluß geben.

\section{Methodik}

Als Analysengut diente die abschabbare Hornschicht des Rückens von 30 mehr als 70 Jahre alten Hautgesunden eines 


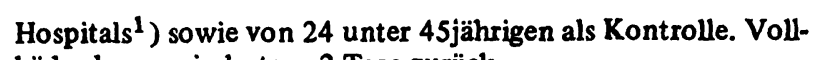
bäder lagen mindestens 2 Tage zurück.

Die wäßrigen Extrakte der Hornschicht-Schabsel (,Wasserlösliches") wurden nach Extraktion mit reichlich Petroläther (Siedepunkt $50-70^{\circ} \mathrm{C}$ ) während $48 \mathrm{~h}$ am Soxhlet, zweimaliger Elution mit dest. Wasser im Verhältnis 1:25 (g/ml) und Zentrifugation bei $3000 \mathrm{~g}$ für $15 \mathrm{~min}$ gewonnen. Aufbewahrung bei $-20^{\circ} \mathrm{C}$.

Aminosäuren, Ammoniak und Harnstoff wurden mit einem automatischen Aminosäuren-Analy sator ${ }^{2}$ ) im Einsäulenverfahren nach 1. c. (37) bestimmt.

Säulenmaß: $54 \cdot 0,9 \mathrm{~cm}, \mathrm{Harz}$ : Aminex A $6^{3}$ )

Puffer: Natrium-Citrat pH 3,25-4,52 $\left(\mathrm{Na}^{+} 0,2-0,8 \mathrm{~mol} / \mathrm{l}\right)$ Durchlaufgeschwindigkeit: $80 \mathrm{ml}$ Puffer $/ \mathrm{h}+30 \mathrm{ml}$ Ninhydrin/h Temperatur: $30^{\circ}, 50^{\circ}$ und $60^{\circ} \mathrm{C}$.

Das Säuleneluat wurde nach Ninhydrin-Färbung bei 570 und $440 \mathrm{~nm}$ gemessen. Als innerer Standard diente Norleucin.

Pyrrolidoncarbonsäure wurde nach quantitativer Hydrolyse zu Glutaminsäure mit $\mathrm{HCl}$ (Endkonzentration $2 \mathrm{~mol} / 1$ ) in einer geschlossenen Ampulle unter Stickstoff $2 \mathrm{~h}$ bei $100^{\circ} \mathrm{C}$ aus dem Zuwachs an Glutaminsäure bei Aminosäure-Analyse ermittelt.

Urocaninsäure wurde mittels Isotopen-Verdünnungs-Analyse (38) und Fotometrie $\left(E_{268}(0,1 \mathrm{~mol} / 1 \mathrm{HCl})\right)$ wie früher beschrieben (39) bestimmt.

Die Lactat-Bestimmung erfolgte nach 1 . c. (40) mit LactatDehydrogenase $\left.{ }^{4}\right)$ anhand der NADH-Bildung $\left(E_{340}\right)$.

Statistische Prüfungen wurden nach 1. c. (41) durchgeführt.

\section{Ergebnisse}

In der Tabelle 1 sind die Mittelwerte und Standardabweichungen der Summe der freien Aminosäuren, bezogen auf Hornschicht-Schabsel-Frischgewicht, sowie die Werte der einzelnen Aminosäuren, von Urea, Ammoniak, Urocaninsäure, Pyrrolidoncarbonsäure und Lactat beider Altersgruppen in $\mathrm{mol} / 100 \mathrm{~mol} \mathrm{Ami-}$ nosäuren angegeben. Die drei letztgenannten Substanzen konnten nicht bei allen Probanden bestimmt werden. Unter der Rubrik X sind unbekannte Ninhydrin-Peaks zusammengefaßt worden, die mengenmäßig nicht ins Gewicht fallen $(<1,0 \mathrm{~mol} \%)$ und bei denen wegen der Heterogenität die Angabe einer Standardabweichung entfiel.

In der Altersgruppe fand sich eine signifikante Vermehrung der freien Aminosäuren insgesamt $(p<0,01)$, von Gly, Val $(p<0,01)$ und Ala $(p<0,05)$ sowie eine Verminderung von Thr, Glu, His, Urea, Lactat $(p<0,01)$ und Citrullin $(p<0,05)$. Die Vermehrung von Urocaninsäure im Alters-Wasserlöslichen erwies sich entgegen früheren Befunden (36), möglicherweise wegen der gröBeren Streuwerte besonders beim Kontroll-Kollektiv, als nicht signifikant.

In der Tabelle 2 sind Korrelationskoeffizienten und Bestimmtheitsmaße (in Prozent) für mehrere Wasserlöslichen-Komponenten angegeben. Strenge Korrelationen $(2 \alpha<0,05)$ in beiden Altersgruppen lagen für Urea/ Lactat, Val/Leu und His/Urocaninsäure vor, lediglich

\footnotetext{
1) Krankenhaus Am Mariendorfer Weg, Berlin, Abt. für Chronisch Kranke (Chefarzt: Prof. Dr. H. Zapfe)

2) BC 200, BIO CAL GmbH, München

3) BIO-RAD-Laboratories München 4) Biochemica-Test-Combination Nr. 159 72, Boehringer,
Mannheim
}

Tab. 1. Mittelwerte und Standardabweichungen (in Klammern, \% von $\overline{\mathbf{x}}$ ) der freien Aminosäuren, von Urea, Ammoniak, Urocaninsäure, Pyrrolidoncarbonsäure und Lactat wäßriger Extrakte abschabbarer Hornschicht alter und junger Hautgesunder.

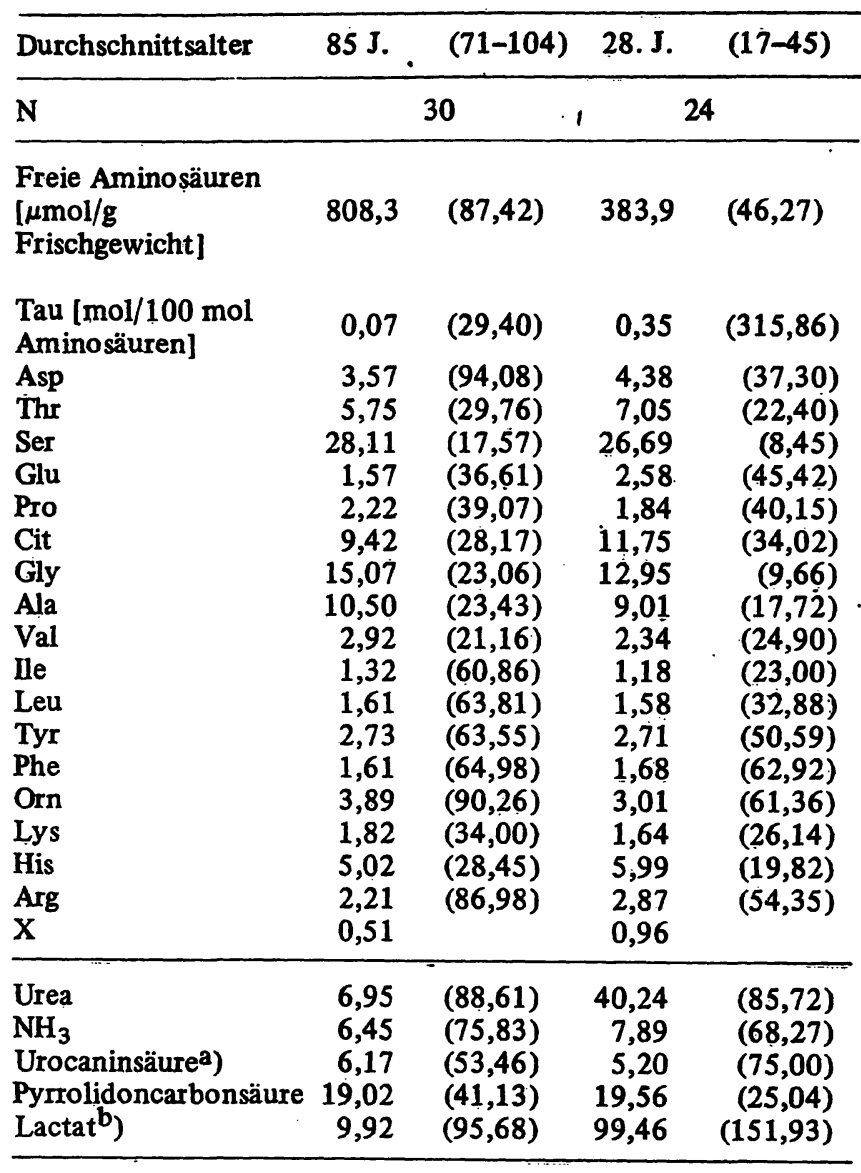

a) $\mathrm{N}=24$ (alt); $\mathrm{N}=18$ (jung)

b) $\mathrm{N}=13$ (alt); $\mathrm{N}=12$ (jung)

in der Altersgruppe für Orn/Cit, Arg/Urea und Leu/Lys sowie lediglich in der Kontrollgruppe für Aminosäuren/ Lactat, Aminosäuren/Urea, Asp/Arg, Gly/Ala und Gly/ Ser.

\section{Diskussion}

Die beobachtete Vermehrung der freien Aminosäuren insgesamt in der Hornschicht der Altershaut steht zwar in Einklang mit früheren Befunden anderer Autoren (42), doch sollten wegen der Abhängigkeit dieser Größe von der zurückliegenden Zeit des letzten Bades (43) auch die besonderen Lebensbedingungen der Altersgruppe in einem Hospital in Betracht gezogen werden.

Der Rückgang der Talgproduktion im Alter infolge reduzierter Testosteron-Bildung führt naturgemäß auch zu einer relativen Vermehrung des Wasserlöslichen, bezogen auf HornschichtSchabsel-Frischgewicht, und damit der freien Amiṇosäuren Andererseits müßte der Talgverlust an der Hautoberfläche alter Leute der leichteren Auslaugbarkeit dẹs Wasserlöslichen durch Waschmaßnahmen Vorschub leisten.

Der Gehalt an freien Aminosäuren in normaler Hornschicht (Schabsel) wird von anderen Untersuchern mit 
Tab. 2. Korrelationskoeffizient $(r)$ und Bestimmtheitsmaß $\left(x^{2}\right)$ verschiedener Komponenten des „Wasserlöslichen " der beiden Altersgruppen UCS = Urocaninsäure, PCS $=$ Pyrrolidoncarbonsäure

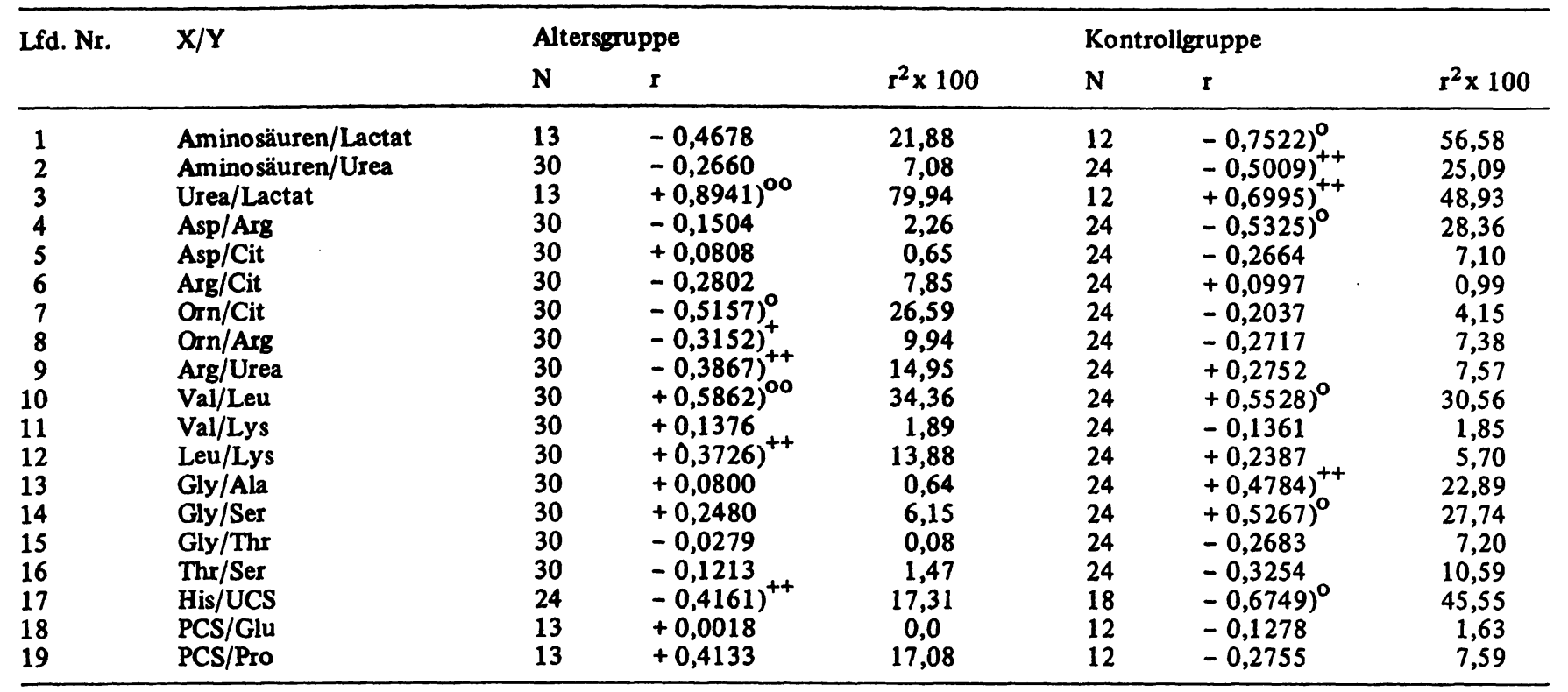

$+{ }_{+}^{+}$Null-Hypothese von $\mathrm{r}$ zu verwerfen für $2 \alpha=0,1 ;$ Freiheitsgrad $=\mathrm{N}-2$

+ )d to. für $2 \alpha=0,05$

ob dto. für $2 \alpha=0,01$

ddto. für $2 \alpha=0,001$

etwa $900(550-1300) \mu \mathrm{mol} / \mathrm{g}$ Frischgewicht (5), für menschliche Gesamt-Epidermis mit $200 \pm 86 \mu \mathrm{mol} / \mathrm{g}$ (44) angegeben. Die Tatsache, daß die Werte für Hornschicht stets um das Doppelte und mehr höher liegen als für Epidermis weist darauf hin, daß das Gros der hier untersuchten Substanzen in ihrem verhornten Anteil liegt.

Die auffäligsten Unterschiede im Wasserlöslichen der beiden Altersgruppen fanden sich offensichtlich für Harnstoff und Lactat, die bei großen individuellen Schwankungen im Alter deutlich reduziert sind.

Lactat kann als ausschließlich sudorigener Bestandteil des Wasserlöslichen betrachtet werden, der nach dem Salinen-Prinzip in der Hautoberfläche abgelagert wird (5).

Für Harnstoff, zweifellos auch Schweißbestandteil $(5,45)$, ist die Frage nach einer zumindest partiell epidermogenen Herkunft nicht eindeutig entschieden, zumal die sog. epidermale Barriere, also die sicherlich nicht mit Schweiß kontaminierten tiefen Anteile der. Hornschicht etwa den gleichen Gehalt aufweisen sollen wie die Peripherie (46). Die enge Korrelation zwischen Urea- und Lactat-Gehalt bei den vorgelegten Untersuchungen, die besonders in der Altersgruppe erkennbar war, (lfd. Nr. 3, Tab. 2) läßt aber wohl den Schluß zu, daß die Minderung von Harnstoff wie die von Lactat auf eine geringere Schweißsekretion im höheren Alter zurückzuführen ist.

In Sommer- resp. Wintermonaten von Hautgesunden gewonnenes Wasserlösliches (Handschuh-Eluate (5)) wies offensichtlich gemäß der jeweiligen Saison-bedingten Schweißrate in Bezug zum Aminọsäuren-Gehalt relativ hohe resp. nịedrige
Lactat-Werte auf. Ungeachtet der saisonalen Verschiedenheiten soll aber eine individuell enge Korrelation zwischen Aminosäuren- und Lactat-Gehalt vorgelegen haben, so daß in Analogie zum talgfördernden auch ein „Aminosäuren-fördernder" Schweißeffekt erwogen wurde (5), der aber mit unseren Ergebnissen nicht in Einklang stünde (negative Korrelation: lfd. Nr.1, Tab. 2).

Wie aufgrund der engen Korrelation zwischen Lactat und Harnstoff zu erwarten war, fand sich bei unseren Untersuchungen gleichermaßen eine umgekehrte Proportionalität zwischen Aminosäuren- und HarnstoffGehalt des Wasserlöslichen (lfd. Nr. 2, Tab. 2), nicht signifikant allerdings bei der Altersgruppe, bei der der anscheinend hierfür verantwortliche sudorigene UreaAnteil weniger ins Gewicht fiel.

Aus vergleichbaren Untersuchungen (5) an normalen Hornschicht-Schabsel lassen sich für Milchsäure resp. Harnstoff 46 resp. $23 \mathrm{~mol} / 100 \mathrm{~mol}$ Aminosäuren errechnen, also jeweils etwa halb so große Werte wie die unserer Kontrollgruppe, obwohl die Relation Lactat: Urea $\sim 2: 1$ ebenfalls erkennbar war. Die Reduktion der fraglichen Werte im höheren Alter wurde von (47) nicht beobachtet.

Die große Variationsbreite der Ergebnisse verschiedener Autoren unterstreicht eigentlich nur die vornehmlich sudorigene Natur von Lactat und Urea. Der Schwankungsbereich der Milchsäure-Konzentration im Schweiß beträgt beispielsweise bis $5,5-55,0 \mathrm{mmol} / \mathrm{l}(5)$. Hingegen lassen sicher epidermogene Substanzen wie die freien Aminosäuren meist gute Übereinstimmung erkennen. Der kalkulierte Wert für Serin aus (5), einer mengenmäßig im Wasserlöslichen prävalierenden Amino- 
säure, entsprach z. B. mit $27,5 \mathrm{~mol} / 100 \mathrm{~mol}$ Aminosäuren durchaus dem unserer Kontrollgruppe.

Die Bedeutung eines erheblich reduzierten Lactat-Gehaltes an der Hautoberfläche für Funktionseinschränkungen im Alter sollte angesichts ihres Stellwertes für „Säuremantel“ und Hygroskopizität nicht unterschätzt werden, wenn auch das ,pH-Problem der Hautoberfläche nicht von der isolierten Betrachtung eines einzelnen, noch so markanten Bestandteils wie etwa der Milchsäure her angegangen werden kann" (5).

Keine Unterschiede zeigten sich bei den Untersuchungsgruppen hinsichtlich der anderen, wesentlich zum Anionen-Anteil (-Überschuß) des Wasserlöslichen beisteuernden Komponente, der Pyrrolidoncarbonsäure. Der von uns gefundene Gehalt von etwa $20 \mathrm{~mol} / 100 \mathrm{~mol} \mathrm{Ami-}$ nosäuren entspricht früheren vergleichbaren Ergebnissen anderer Autoren (5). Pyrrolidoncarbonsäure fand sich auch in der (Gesamt-) Epidermis des Menschen und mehrerer Säugetiere $(44,48)$. Unlängst konnte sie als anscheinend normaler Plasma-Bestandteil beim Menschen und Meerschweinchen in Konzentrationen von 0,22 bzw. $0,33 \mathrm{mmol} / 1$ nachgewiesen werden (49).

Pyrrolidoncarbonsäure wird in der Epidermis, offenbar fermentativ katalysiert (50), durch Dehydration von Glutaminsäure (4) gebildet. Auch eine oxydative Entstehung aus Prolin wird erwogen (4). Eine umgekehrte Proportionalität zwischen Pyrrolidoncarbonsäure und den genannten Verbindungen, die auf ein Praecursor-Produkt-Verhältnis hätte schließen lassen, war nicht erkennbar (ffd. Nr. 18 u. 19, Tab. 2).

Wegen der offensichtlichen Autoregulation des „Säuremantels" auch bei stark wechselnden Schweißraten wird dem Reservoir epidermogener saurer Valenzen in den tiefen Hornschichtlagen (Barriere) größeres Gewicht beigemessen (5). Als Substrat desselben dürfte neben Asparagin- und Glutaminsäure vor allem Pyrrolidoncarbonsäure gelten. In der Tat würde die Beobachtung, daß das aktuelle Oberflächen-pH der Altershaut trotz reduzierter Lactat-Werte eher zum Sauren tendiert (51), für die Priorität epidermogener Säure-Valenzen sprechen.

Pyrrolidoncarbonsäure dürfte an der Hautoberfläche zu über 95\% als Pyrrolidonat vorliegen (5). Da die Salze stark hygroskopisch sind, scheint ihnen ähnlich wie Milchsäure für die normale Hautbeschaffenheit eine besondere Rolle zuzukommen.

Die gefundene Vermehrung von Gly, Ala und Val in der Alters-Hornschicht ist schwer zu deuten. Parallelen zu Befunden an pathologischen Verhornungsprodukten bestehen nicht.

Typisch für bestimmte Epidermis-Proteine ist ihr GlyReichtum mit bis $33,8 \mathrm{~mol} / 100 \mathrm{~mol}$ Aminosäure (22), die anscheinend vorzugsweise in den oberen Zellagen der lebenden Epidermis, dem Strat. granulosum, gebildet werden und in der peripheren Hornschicht einer (zumindest partiellen) Auflösung unterworfen zum Pool der freien Aminosäuren des Wasserlöslichen beitragen.

Die Korrelation der Gly-Werte mit Ala und Ser (lfd. Nr. 13 u. 14, Tab. 2), die nur bei der Kontrollgruppe zu eruieren war, ließe vermuten, daß diese Aminosäuren ebenfalls Komponenten der betreffenden Proteine sind. Ser weist in der Tat das gleiche Einbau-Maximum wie Gly auf $(20,21)$, für Ala ist es nicht bekannt. Die fehlende Korrelation der fraglichen Aminosäuren-Werte in der Alters-Hornschicht könnte demnach auf eine diesbezügliche Protein-Aberration hinweisen.

Die histoautoradiographisch feststellbare Úbereinstimmung hinsichtlich von Inkorporierungs-Maxima żweier oder mehrer Aminosäuren impliziert aber wohl nicht ohne weiteres eine Beteiligung am Aufbau gleicher Proteine oder gleicher Teilstücke derselben. Liegen jedoch gleichzeitig Korrelationen der fraglichen Aminosäuren-Werte im Wasserlöslichen vor, ist eine solche Deutung eher möglich, fehlende Korrelationen dürften aber kaum überraschen.

Val ist im Gegensatz zu den vorgenannten Aminosäuren als bevorzugter Baustein der im Strat. basale synthetisierten Proteine und demzufolge $u$. a. auch der Tonofibrillen (-filamente) anzusehen. Für Aminosäuren mit gleichem Einbau-Schwerpunkt wie etwa Leu ergab sich auch eine ziemlich strenge Korrelation in beiden untersuchten Gruppen (lfd. Nr. 10, Tab. 2), zu anderen wie etwa Lys nicht (lfd. Nr. 11, Tab. 2).

Die Verminderung von Thr, Glu, His und Cit in der Altershornschicht kann ebenfalls nicht eindeutig interpretiert werden.

Thr weist an sich unter den freien Aminosäuren normaler Hornschicht wie auch pathologischer Verhornungsprodukte relativ konstante Werte auf. Korrelationen zu Gly oder Ser ließen sich nicht feststellen (lfd. Nr. 15 u. 16, Tab. 2). Uber eine besondere Rolle im Epidermis-Stoffwechsel ist nichts bekannt.

Glu ist ein mengenmäßig wenig ins Gewicht fallender Bestandteil des Wasserlöslichen. Auf vermutliche Beziehungen zum Pyrrolidoncarbonsäure-Gehalt wurde eingangs hingewiesen.

His ist im Gegensatz zum Befund bei Altershaut im Wasserlöslichen klinisch unauffälliger Haut von mikrobiellen Ekzematikern (34) und Neurodermitikern (33) gegenüber der Norm erhöht gefunden worden.

Als Quelle für His im Wasserlöslichen ist wohl der (partielle) Abbau verschiedener Epidermis-Proteine anzusehen. Ein Hisreiches Protein $(22,23,52)$ ist Kemponente des Keratohyalins $(53,54)$ und mit der Bildung von Urocaninsäure verbunden (55). Fehlt Keratohyalin wie bei psoriatischer Parakteratose, fehlt auch Urocaninsäure $(7,10)$. Histidinaemie-Kranke sollen trotz vorhandenen Keratohyalins in der Epidermis keine Urocaninsäure aufweisen (56). Für ein Praecursor-Produkt-Verhältnis zwischen His und Urocaninsäure in der Epidermis (57) sprechen die negativen Korrelationskoeffizienten (lfd. Nr. 17, Tab. 2).

Cit ist ebenso wie in der Alters-Hornschicht im Wasserlöslichen verschiedener pathologischer Verhornungsprodukte wie Psoriasis-Schuppen (31) und Callus (32) aber auch der palmo-plantaren Hornschicht (9) vermindert gefunden worden, anscheinend bevorzugt bei ,kompakten" Verhornungstypen mit verzögerter Abstoßung der Hornzellen.

Cit ist ungewöhnlicher Protein-Bestandteil der inneren Wurzelscheiden und Medullae von Haaren bzw. Äquivalenten (58, 59) und anscheinend auch der Epidermis (60), offenbar vor- 
wiegend in keratinisierenden Geweben, die im Gegensatz etwa zu Haaren oder Nägeln einer späteren Auflösung unterliegen. Cit auch als Metabolit des Harnstoff-Zyklus zu betrachten, ist naheliegend, doch wird die Funktion desselben in der Epidermis bestritten (61). Der histochemische Nachweis von Arginase
$(62,63)$ zeigt auffällige Ubereinstimmung mit der Lokalisation Cit-haltiger Proteine.

Negative Korrelationen zwischen Aminosäuren, die am Harnstoff-Zyklus beteiligt sind, fanden sich wenn auch unregelmäßig, fast nur in der Altershaut (lfd. Nr. 4, 5, 6, 7, 8 u. 9, Tab. 2).

\section{Literatur}

1. Spier, H. W. \& Pascher, G. (1955), Arch. Derm. Syph. 199, 411-427.

2. Spier, H. W. \& Pascher, G. (1955), Arch. Klin. Exp. Dermatol. 200, 59-66.

3. Spier, H. W. \& Pascher, G. (1956), Der Hautarzt, 7, 55-60.

4. Spier, H. W. \& Pascher, G. (1957), Acta Dermatol. Ven. Proc. IIth Int. Congr. Dermat. II, 14-22.

5. Spier, H. W. \& Pascher, G. (1959), Akt. Probl. Dermatol. 1, $1-46$.

6. Spier, H. W. \& Schwarz, E. (1962), Proc. XII. Inter. Congr. Dermatol. Washington.

7. Schwarz, E. (1966), Arch. Klin. Exp. Dermatol. 227, 191202.

8. Schwarz, E. (1967), Z. Haut-Geschl.-Krkh. 42, 791-806.

9. Burke, R. C., Lee, Th. \& Buettner-Janusch, V. (1966), Yale J. Biol. Med. 38, 355-373.

10. Schwarz, E. (1967), diese Z. 5, 247-251.

11. Märchionini, A. \& Spier, H. W. (1959), in Handbuch der allgemeinen Pathologie (Hrsg. Büchner-Lekerer-Roulet) Bd. V/2, S. 527-600. Springer-Verlag, Berlin-GöttingenHeidelberg.

12. Szakall, A. (1955), Arch. Klin. Exp. Dermatol. 201, 331360.

13. Dowling, G. B. \& Naylor, F. D. (1960), Brit. J. Dermatol. 72, $51-56$.

14. Spier, H. W. \& Beiersdorff, H. U. (1964), Arch. Klin. Exp. Dermatol. 219, 613-619.

15. Szakall, A. (1957), Arch, Klin. Exp. Dermatol. 206, 374379.

16. Röckl, H., Spier, H. W. \& Pascher, G. (1957), Arch. Klin. Exp. Dermatol. 205, 420-434.

17. Miescher, G. \& Speck, K. (1957), Naunyn-Schmiedebergs Arch. Pharmakol. Exp. Pathol. 230, 223-227.

18. Block, R. J. (1937), J. Biol. Chem. 127, 761.

19. Rothman, S. (1954), Physiology and Biochemistry of the Skin. The university of Chicago press.

20. Fukuyama, K., Nakamura, T. \& Bernstein, I. A. (1965), Anat. Rec. 152, 525-536.

21. Fukuyama, K. \& Epstein, W. L. (1966), J. Invest. Dermatol. 47, 551-560.

22. Bernstein, I. A. (1970), J. Soc. Cosmet. Chem. 21, 583594.

23. Chakrabarti, S. \& Bernstein, I. A. (1966), Abstracts 152nd Natl. Meeting Amer. Chem. Soc., N. Y.

24. Chakrabarti, S. G. \& Bernstein, I. A. (1967), Fed. Proc. $26,369$.

25. Fukuyama, K. \& Epstein, W. L. (1969), J. Cell. Biol. 40, 830-838.

26. Gillespie, J. M. \& Darskus, R. L. (1972), Vortrag XIV. Int. Cong. Dermatol., Padua-Venedig.

27. Ugel, A. R. (1971), J. Cell. Biol. 49, 405.

28. Pascher, G. (1964), Arch. Klin. Exp. Dermatol. 218, 111125.

29. Matoltsy, A. G. \& Matoltsy, M. N. (1966), J. Invest. Dermatol. 46, 127-129.

30. Crounse, R. G. (1964), The epidermis (ed. Monlagna, W. \& Lobitz, W. G.) Academic Press, pages 365-374.

31. Kloss, G. \& Schwarz, E. (1967), Arch. Klin. Exp. Dermatol. $228,188-198$.

32. Schwarz, E., Thies, W. \& Kloss, G. (1969), Arch. Klin. Exp. Dermatol. 235, 43-52.
33. Schwarz, E. (1968), Vortrag auf der 45. Tagung der Nordwestdeutschen Dermatolog. Gesellschaft gemeinsam mit der Hamburger Dermatolog. Ges., Norderney.

34. Schwarz, E. (1971), Arch. Dermatol. Forsch. 242, 87-96.

35. Schwarz, E. \& Kloss, G. (1968), Arch. Klin. Exp. Dermatol. 231, 311-317.

36. Kügelgen, H. von \& Schwarz, E., Arch. Dermatol. Forsch. im Druck.

37. Piez, K. A. \& Morris, L. (1960), Anal. Biochem. 1, 187201.

38. Radioactive isotope dilution analysis. (1964), 2 nd edition. RCC Review No. 2. Amersham, Radiochemical Centre.

39. Schwarz, E., Witzke, G., Jacob, M. \& Pech, H. (1971), Arch. Dermatol. Forsch. 240, 173-183.

40. Hohorst, H. J. (1970), in: Methoden der enzymatischen Analyse, (Bergmeyer, H. U., Hrsg.) Bd. II, 2. Aufl. S. 1425-1429. Verlag Chemie, Weinheim.

41. Documenta Geigy, Wissenschaftliche Tabellen, Basel 1960.

42. Salfeld, K. (1965), Aesthet. Med. 14, 332-337.

43. Dowling, G. B. \& Naylor, P. F. (1960), Brit. J. Dermatol. $72,57-61$.

44. Wolfersberger, M. G., Tabachnick, J., Finkelstein, B. \& Levin, M. (1973), J. Invest. Dermatol. 60, 278-281.

45. Schwarz, E. (1968), Arch. Phys. Therap. 5, 349-360.

46. Stüpel, H. \& Szakall, A. (1957), Die Wirkung von Waschmitteln auf die Haut, Hüthig, Heidelberg.

47. Jacobi, O. (1971), Arch. Klin. Exp. Dermatol. 240, $107-118$.

48. Tabachnick, J. \& LaBadie, J. H. (1970), J. Invest. Dermatol. 54, 24-31.

49. Wolfersberger, M. G. \& Tabachnick, J. (1973), Experientia 29, 346.

50. Schwarz, E. (1964), Arch. Klin. Exp. Dermatol. 219, 593-598.

51. Salfeld, K. (1965), Aesthet. Med., 14, 313--320.

52. Bernstein, I. A., Chakrabarti, S. G., Kumaroo, K. K. \& Sibrack, L. A. (1970), J. Invest. Dermatol. 55, 291302.

53. Fukuyama, K. \& Epstein, W. L. (1967), J. Invest. Dermatol. 49, 595.

54. Fukuyama, K. \& Epstein, W. L. (1971), J. Invest. Dermatol. 56, 211-222.

55. Schwarz, E. (1970), Arch. Klin. Exp. Dermatol. 237, 675-683.

56. Baden, H. P., Hori, Y., Pathak, M. A. \& Levy, H. L. (1969), Arch. Dermatol. Chic. 100, 432-435.

57. Schwarz, E. (1961), Biochem. Z. 334, 415-424.

58. Rogers, G. E. (1963), J. Histochem. Cytochem. 11, 700705.

59. Rogers, G. E. (1964), in The Epidermis (Montagna, W. \& Lobitz, W. C., jr., ed.), Academic Press New York and London, p. 179-236.

60. Schwarz, E. \& Berger, M. (1973), Arch. Dermatol. Forsch. 246, 167-174.

61. Crounse, R. G. \& Rothberg, S. (1961), J. Invest. Dermatol. $36,287-292$.

62. Rothberg, S. \& van Scott, E. J. (1958), J. Invest. Dermatol. $31,263-268$.

63. Crounse, R. G. \& Rothberg, S. (1960), J. Invest. Dermatol. $35,107-111$. 